\title{
Attenuation Correction for Rotating Slant-Hole (RSH) SPECT using Exact Rebinning
}

\author{
Jean-Marc Wagner, Frédéric Noo, Rolf Clackdoyle, Girish Bal, Paul Christian
}

\begin{abstract}
We have recently derived an analytic method of image reconstruction for the RSH SPECT scanner for cardiac imaging. The method assumes that the emission activity lies in a region of constant attenuation. It operates by rebinning the three-dimensional (3D) measured projections to a stack of two-dimensional (2D) slices and performing $2 \mathrm{D}$ reconstruction in a second step. An important consequence of this method is a mathematical proof that opposing projections are not necessary for full tomographic reconstruction in the presence of attenuation. We have now tested our algorithm in the presence of noisy projection data and with projections which violates the constant attenuation assumption. We present results from simulated and real phantom data demonstrating effective attenuation correction in the presence of a uniform low-level of background in the torso, with truncated liver activity, and without opposing views. Our conclusions are that attenuation correction can be performed for RSH SPECT without measuring opposing views, and that the effect of background activity (which violates the assumptions required for exponential data) and truncated activity of a nearby liver are small and easily dominated by typical noise levels in SPECT imaging.
\end{abstract}

\section{BACKGRound}

A novel RSH SPECT scanner for cardiac imaging is under development at the University of Utah. The main feature is a rotating slant-hole (RSH) collimator with which limited angle tomographic data can be obtained for a fixed position of the detector head. Full tomographic data is acquired by rotating the detector head to a small number of angular positions carefully chosen to satisfy Orlov's condition [1] for 3D parallel projections. The main goal of the RSH SPECT system is improved photon sensitivity which is achieved at the cost of a reduced field-of-view by using multiple segments in the collimator. Multiple segments allow simultaneous collection of several projections, so the expected sensitivity improvement over a conventional parallel-hole collimator is roughly equal to the number of segments, typically 2 or 4 . A similar RSH SPECT system is being developed at the University of North Carolina. In SPECT imaging, quantitative reconstructions can only be achieved if photon attenuation is taken into account. Our RSH SPECT scanner does not have the hardware capability to perform transmission measurements, so alternative techniques for attenuation correction must be considered. We are currently using data consistency conditions in three-dimensions to estimate the attenuation information required for reconstruction of the emission activity [2]. This technique assumes that the emission activity lies in a convex region of constant attenuation $\mu_{c}$, such as a

J.M. Wagner is with the Electricity Montefiore Institute, University of Liege, Belgium. E-mail: JM.Wagner@ulg.ac.be

F. Noo, R. Clackdoyle, G. Bal, P. Christian are with the Medical Imaging Research Laboratory, University of Utah, USA. cylinder enclosing the heart and liver but not intersecting the lungs or spine. The data consistency approach essentially finds attenuation factors which allow conversion of the measured projections to a set of consistent exponential projections $p_{\mu_{c}}(\underline{n},$.$) , which are related to the activity$ distribution $f(\underline{x})$ by

$$
p_{\mu_{c}}(\underline{n}, \underline{s})=\int_{-\infty}^{+\infty} f(\underline{s}+t \underline{n}) e^{\mu_{c} t} d t
$$

where the pair $(\underline{n}, \underline{s})$ specifies a line in space passing through one of the slant holes of the collimator. The projection direction is indicated by the unit vector $\underline{n}$, and $\underline{s}$ which is perpendicular to $\underline{n}$, is the vector from the origin to the nearest point on the line. For the RSH SPECT geometry, the vector $n$ traces out a circle on the unit sphere for each position of the detector gantry. The circle is traced as the collimator rotates, and it has an angular aperture of $2 \alpha$ where $\alpha$ is the fixed slant angle of the collimator. The center of the circle represents the normal to the detector face. For the RSH SPECT geometry, the centers of the circles are spaced by at most $2 \alpha$ and lie on a great circle $C$ whose normal vector corresponds to the gantry rotation axis. See figure 1 for an example.

\section{Principles}

From the exponential projections, we have derived a method of analytic reconstruction for the RSH SPECT geometry, providing the set of projection directions satisfies Orlov's condition. The method is based on a rebinning formula which allows new projections $p_{\mu}$ to be synthesized for any direction $\underline{n}$ lying inside a circle of measured projections. The projection can be synthesized with any attenuation value $\mu$ (including $\mu=0$ ) but the most stable value for the rebinning is $\mu=\mu_{c}$. We have described this rebinning formula in [3]. The method of reconstruction involves rebinning the measured projections to a set of projections lying on the great circle $C$ and therefore synthesizing exponential projections for a conventional parallel-hole collimator system. From there we apply existing techniques to complete the reconstruction. For example, if the rebinned projections cover the whole (360 degrees) of $C$, we could use the two-dimensional reconstruction algorithm of Tretiak and Metz [4] slice-by-slice. We appeal to more general results, such as [5] or [6] to handle the case of a semi-circle (180 degrees) of rebinned projections.

An important consequence of these 180-degree cases is that it establishes that there is no theoretical need for conjugate (diametrically opposing) projection views in the RSH SPECT geometry. One of the purported strengths of 

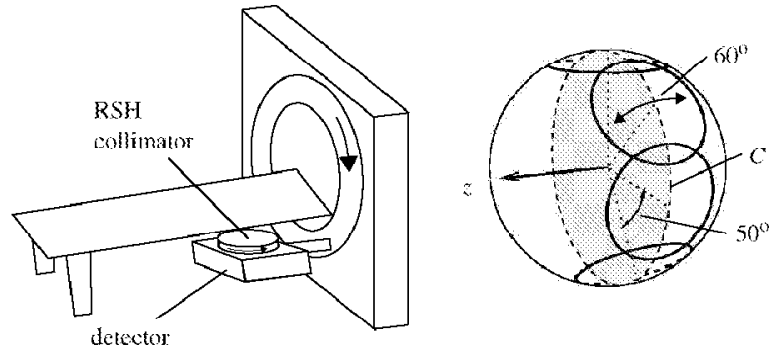

Fig. 1. Illustration of the RSH SPECT scanner (left), and the trace of the projection directions on the unit sphere (right).

the RSH SPECT concept is small number of gantry positions, and this theoretical result halves the required number.

\section{Simulations}

We present the results of simulations and real phantom experiments which indicate that (i) the algorithm can produce effective attenuation correction with real data; (ii) the assumption of a constant attenuation region can be violated to some extent; and (iii) that truncated activity from nearby organs such as the liver does not produce significant artifacts.

The simulated and phantom geometries were similar. The slant angle of the collimator was $\alpha=30$ degrees. The detector was moved in steps of 50 degrees between four locations on the left side of the body, so the circles on the unit sphere overlapped as shown in figure 1. The total angular range of motion was 150 degrees at angles 20,70,120, 170 degrees LAO.
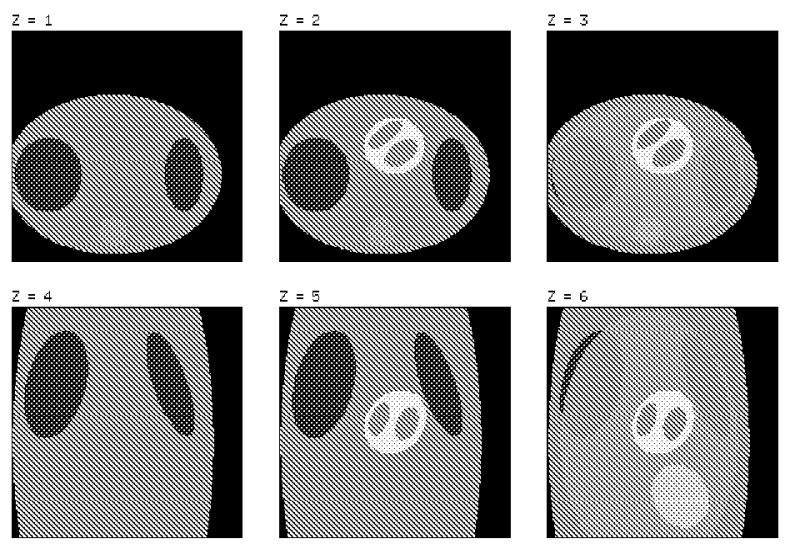

Fig. 2. Attenuation map (left); attenuation with heart activity superimposed (middle); attenuation with heart, liver, background (right).

For the computer simulations, a mock heart was built from 3 ellipsoids with activity levels of 1.0 in the heart wall, and 0.2 in each of the two ellipsoidal ventricles. A small ellipsoidal liver (the same volume as the heart) was also simulated with a uniform activity level of 0.6. The total activity in the liver was $80 \%$ of the total in the heart. A large ellipsoid of background activity was also simulated whose total activity equaled that of the heart, resulting in a specific activity of 0.02 . The attenuation distribution was modeled using ellipsoids for the torso, lungs, and spinal cord with assigned values of $0.152 / \mathrm{cm}, 0.039 / \mathrm{cm}$, and $0.248 / \mathrm{cm}$ respectively. Figure 2 illustrates the geomctry of the attenuation and emission activities.

Attenuated projections were simulated of size $100 \times 100$ pixels and side $1.5 \mathrm{~mm}$ at each of 32 angular positions of the collimator in steps of 11.25 degrees. All 128 projections were centered on the simulated heart. The projection values were obtained using exact line-length calculations through the ellipsoids and applying the attenuation factors analytically. Four experiments were performed, corresponding to simulations of (a) the heart only, (b) the heart with Poisson noise added corresponding to 200,000 counts in each projection, (c) the heart with liver and background but no noise, and (d) the heart, liver and background with noise added at the level of 200,000 counts per projection. Figure 3 shows some typical simulated projections.
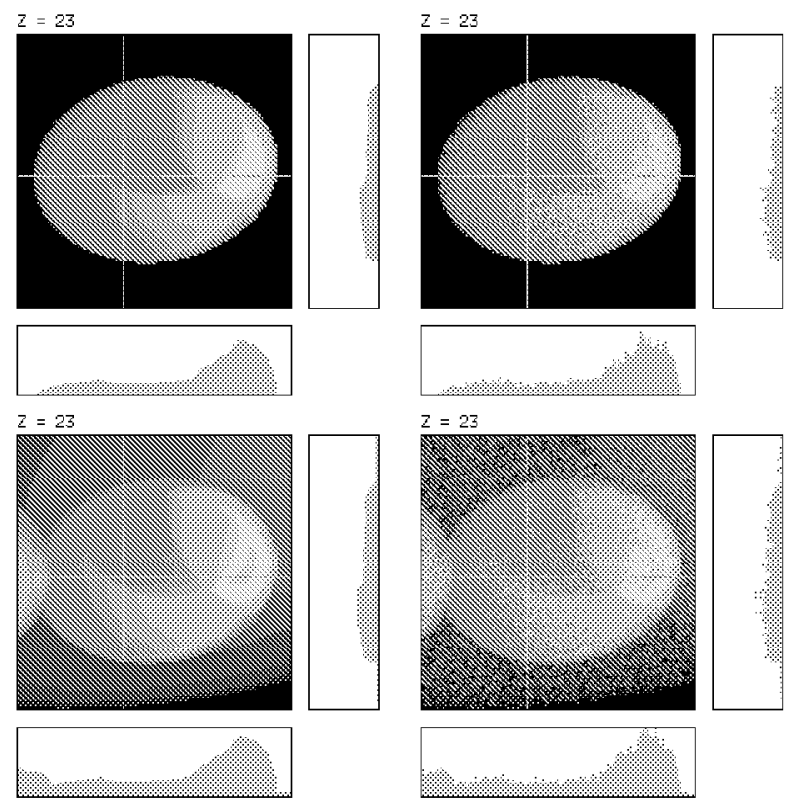

Fig. 3. Simulated projections. (a) heart only (top left); (b) heart with noise (top right); (c) heart with liver and background (bottom left); (d) heart with liver, background and noise (bottom right).

The data were processed as follows. First, the 128 simulated projections were converted using standard point-bypoint multiplication [7] to exponential projections (using $\mu_{c}=0.152 / \mathrm{cm}$ ). The multiplicative factors were obtained from knowledge of the attenuation distribution. Then, using the rebinning formula, 121 exponential projections were synthesized at 1.5 degree steps along the great circle $C$, giving a range of 180 degrees centered on the 210 degrees spanned by the 4 circles. These projections were the same dimensions and size as the measured projections. The central 107 synthesized projections used $\mu=\mu_{c}$ and the 7 projections at each end used values of $\mu$ which ramped down to zero for the 1st and 121st synthesized exponential projections. From these 121 synthesized projections, 2D reconstructions were performed for 100 slices in $z$ (the scanner axis) using a version of the Tretiak and Metz FBP 
algorithm, modified according to [5] to handle the angularly varying $\mu$. Each slice was size $100 \times 100$, with a slice separation of $1.5 \mathrm{~mm}$ and pixel size $1.5 \mathrm{~mm}$.

Real data was measured using our prototype RSH SPECT scanner at the University of Utah (with thanks to D. Bone, H. Elmqvist, S. Dale of the Karolinska Institute, Stockholm for the loan of the 2-segment RSH collimator). The standard Data Spectrum cardiac insert with a single wall defect was filled with activity and placed inside a hollow anthropomorphic hollow torso. Projections of size $256 \times 256$ were acquired with a detector pixel size of $2.33 \mathrm{~mm}$. The collimator was rotated in steps of 11.25 degrees for a total of 32 projections per detector position. The four positions of the gantry were separated by steps of 50 degrees and traversed the left side of the phantom as described above. The total number of projections was 128. Since this phantom was in air, the rebinning step synthesized 181 unattenuated $(\mu=0)$ projections of size 80 $\mathrm{x} 80$ pixels of side $2 \mathrm{~mm}$ and centered on the projection of the cardiac insert. These data were reconstructed using standard 2D FBP (no attenuation) for 80 slices in $z$. Each slice was $80 \times 80$ pixels with pixel size and slice separation both equal to $2 \mathrm{~mm}$.

A second phantom experiment was performed with the same imaging parameters except that the torso shell was filled with water, to give a nearly uniform attenuation distribution. The first step of the data processing involved the method of [2] to find the attenuation geometry and constant attenuation coefficient $\mu_{c}$. Exponential data were obtained from the measured projections, with an effective attenuation value of $\mu_{c}=0.12 / \mathrm{cm}$. These exponential data were also rebinned to 181 projections spaced by 1 degree along the circle $C$, and with $\mu=\mu_{c}$ except for ramping down to $\mu=0$ over the first and last 10 projections. The reconstruction was performed using the same algorithm as for the computer-simulated data but using the $80 \times 80 \times$ 80 geometry of the unattenuated phantom reconstruction.

To see the effect of uncorrected attenuation, the second phantom experiment was also processed as if no attenuation were present - using the identical steps to the first phantom reconstruction.

\section{Results And Conclusions}

The four rows of figure 4 show two slices through the phantom for each of the four simulated data sets. Figure 5 shows reconstruction of the phantom data. We immediately observe that the reconstruction from the ideal isolated noise-free heart data (experiment (a)) shows excellent uniformity and no sign of attenuation artifacts. When noise is added to the data, the resulting reconstruction images are noisier as expected. When liver and background activity are added, they cause only barely discernible effects which are only visible on close comparison to the ideal heart reconstructions. When noise is added to these images, it completely dominates. Our conclusions are that effective attenuation correction can be performed for RSH SPECT without measuring opposing views, and that the effects of background activity (which violates the assumptions re-
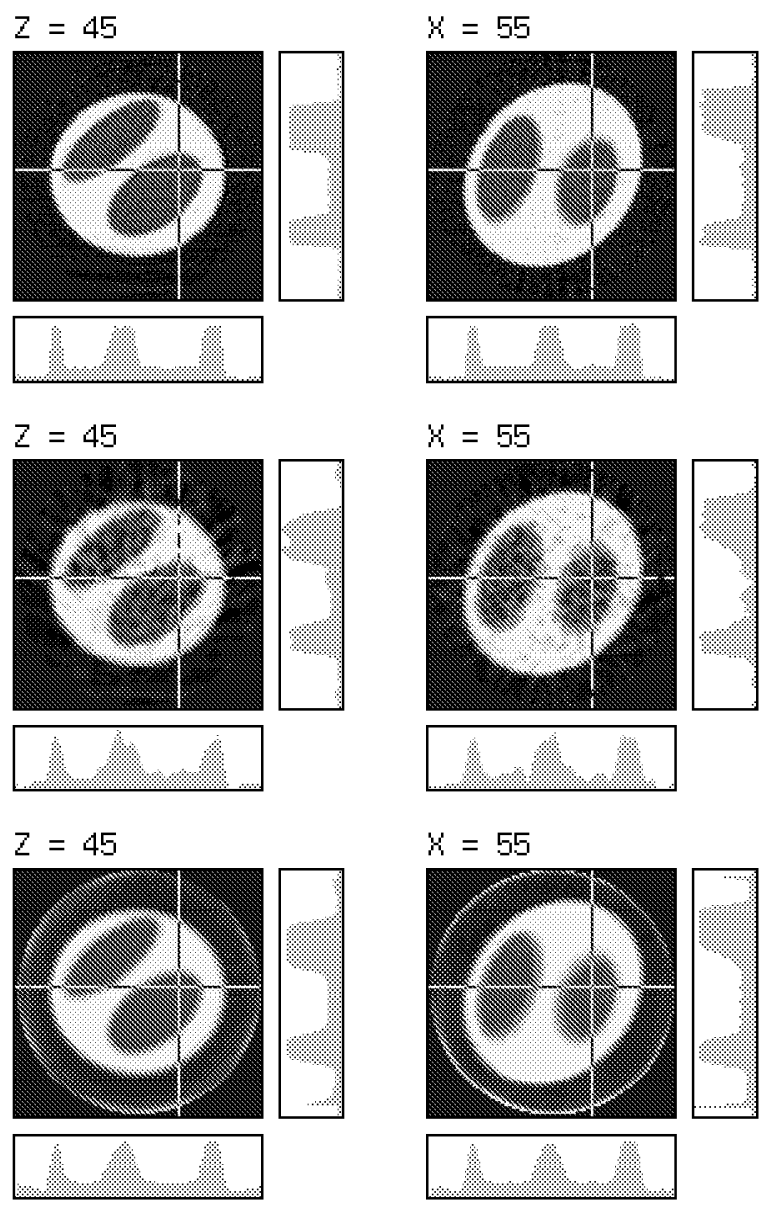

$x=55$
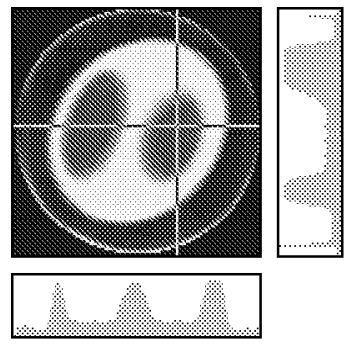

$z=45$
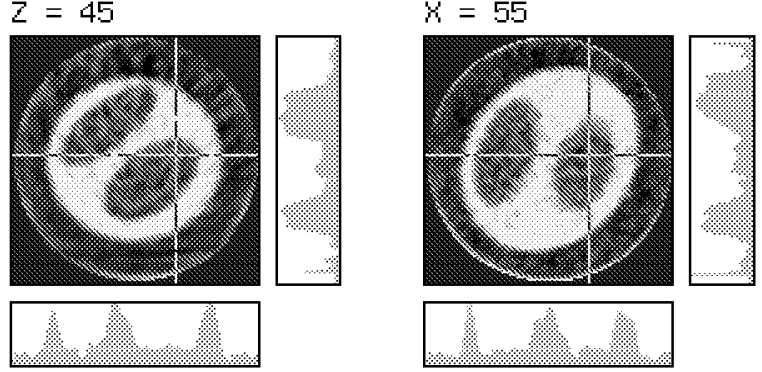

Fig. 4. Attenuation-corrected reconstructions from simulated data. (a) heart only (top row); (b) heart with noise (2nd row); (c) heart with liver and background (3rd row); (d) heart with liver, background and noise (bottom).

quired for exponential data) and nearby truncated activity of the liver are small and easily dominated by typical noise levels in SPECT imaging. The bright ring is a minor artifact occuring at the edge of the reconstructed imaging volume, easily removed if necessary by extending the projection size before applying 2D FBP.

The top row of figure 5 shows three slices through the reconstruction of the heart in air. The walls show good uniformity which suggested that the program was working correctly for the case $\mu_{c}=0$. This code was run for the data gathered in the presence of the attenuation medium to give a control case - uncorrected attenuation. The images in the middle row show the usual effects of atten- 


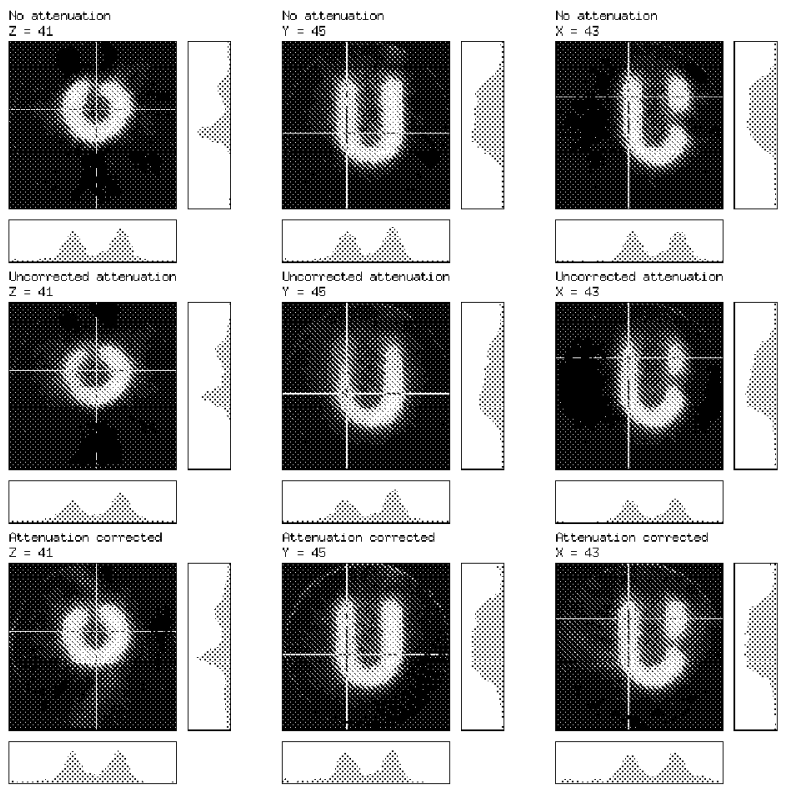

Fig. 5. Resuts from phantom experiments. Reconstruction of heart phantom in air (top row); reconstruction of attenuated heart phantom, but not applying attenuation correction (middle row); reconstruction with attenuation correction (bottom row).

uation with apparent decreased intensity away from the apex. The third row, showing the reconstruction using the attenuation procedure described here appears to correctly compensatc for attcnuation, and produccs a reconstruction of very similar quality to the top row when unattenuated data was acquired. We conclude that the rebinning method produces good attenuation correction in phantom images.

\section{ACKNOWNLEDGMENT}

This work was partially supported by the National Institutes of Health, grant number R01 HL55610. Thanks also to H.Elmqvist, D.Bone, S.Dale of the Karolinska Institute and Karolinska Hospital, Stockholm, Sweden.

\section{REFERENCES}

[1] S.S. Orlov, "Theory of three dimensional image reconstruction. 1. Conditions for a complete set of projections." Sov. Phys.Crystallogr., 20, 312-314, 1975.

[2] C. Mennessier, F. Noo, R. Clackdoyle, G. Bal, L. Desbat, "Attenuation correction in SPECT using consistency conditions for the exponential ray transform." Phys. Med. Biol., 44, 2483-2510, 1999.

[3] J.-M. Wagner, F. Noo, R Clackdoyle, "Exact inversion of the exponential x-ray transform for RSH SPECT", submitted to the 2001 International Meeting on Fully Three-Dimensional Image Reconstruction in Radiology and Nuclear Medicine. April 2001.

[4] O. Tretiak, C. Metz, "The exponential Radon transform.", SIAM J. Appl. Math. 39, 341-354, 1980.

[5] P. Kuchment, I Shneiberg, "Some inversion formulas in Single Photon Emission Computed Tomography", Appl. Anal. 53, 221$231,1994$.

[6] F. Noo, J.-M. Wagner, "Image reconstruction in 2D SPECT with 180-degree acquisition", submitted to Inverse Problems. January 2001.

[7] A. Markoe. "Fourier inversion of the attenuated X-ray transform", SIAM J. Math. Anal. 15(4), 718-722, 1984. 